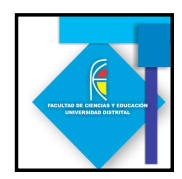

\title{
¿A QUIÉN SE LE ENSEÑA FÍSICA MODERNA?, UNA MIRADA EN INSTITUCIONES EDUCATIVAS DE BOGOTÁ-COLOMBIA
}

\author{
WHO DO YOU TEACH MODERN PHYSICS?, A LOOK AT \\ EDUCATIONAL INSTITUTIONS IN BOGOTA-COLOMBIA
}

Ana Milena Prieto ${ }^{1}$

Orlando Organista ${ }^{2}$

\section{Resumen}

Se presentan datos relacionados con la enseñanza de la física moderna en las carreras de ingeniería en instituciones universitarias de Bogotá. Estos datos son interpretados con el fin de abordar la pregunta ¿qué difusión tiene la física moderna a nivel curricular en la formación profesional de los ingenieros?; este diagnóstico nos llevará a proponer un conjunto de reflexiones alrededor de la enseñanza o no de las ideas modernas de la física en este contexto.

Palabras clave: Enseñanza de la física, física moderna, programas académicos, estudiantes de ingeniería

\begin{abstract}
There is data related to the teaching of modern physics in engineering careers in institutions of Bogotá. These data are interpreted in order to approach the question; what is the diffusion of modern physics in a curricular level, in the professional formation of engineers? .This diagnostic will take us to propose different reflections around the teaching or not of modern ideas of physics in this context.
\end{abstract}

Keywords: teaching of physics, modern physics, academic programs, engineering students.

\section{Introducción}

La presente investigación busca conocer el estado de la enseñanza de la física moderna en instituciones universitarias de Bogotá que ofrecen programas de ingeniería, con el fin de saber qué difusión tienen estas teorías modernas a nivel curricular; este diagnóstico nos llevará a proponer un conjunto de reflexiones alrededor de la enseñanza o no de las ideas modernas de la física en este contexto.

${ }^{1}$ Universidada Pedagógica Nacional, Bogotá, milvenus@hotmail.com

${ }^{2}$ Universidad Central: Física y Matemática, jorganistar@ucentral.edu.co 


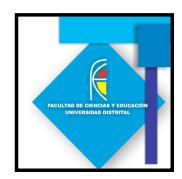

Para conocer el estado de la enseñanza de la física moderna partimos de identificar el porcentaje de universidades que ofrecen carreras de ingeniería, luego se muestra el porcentaje de los programas que en su plan de estudio ven algún tópico de física moderna y finalmente, se diferencia los programas de ingeniería que requieren temáticas modernas de la física.

\section{La Enseñanza de la Física Moderna en Estudiantes de Ingeniería}

Puesto que nuestro interés es la difusión de la física moderna en estudiantes de ingeniería, se requiere conocer el número de instituciones que ofrecen carreras de ingeniería. Está información se obtiene revisando la base de datos de dos entidades gubernamentales que suministran la información confiable, oportuna y relevante para este estudio, El Sistema Nacional de Información de la Educación Superior (SNIES) y La Unidad Administrativa Especial de Catastro Distrital. Se encontró un total de 111 instituciones universitarias, de las cuales 42 tiene algún programa de ingeniería, figura1.

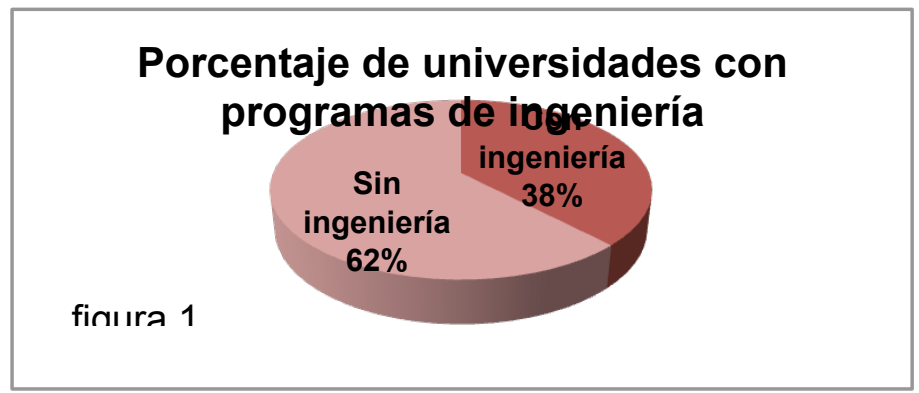

Figura 1

Asumiendo que la física es básica en la formación de un ingeniero se esperaría que este $38 \%$ de estudiantes recibieran un curso con tópicos de física moderna. Como veremos, no es el caso. De las 42 universidades que ofrecen programas de ingeniería se observa que 19 de estas instituciones tienen en su plan de estudios algún tópico de física moderna, así del total de las carreras de ingeniería solo en el $17 \%$ hay una difusión de la física moderna; esta información se sintetiza en la figura 2.

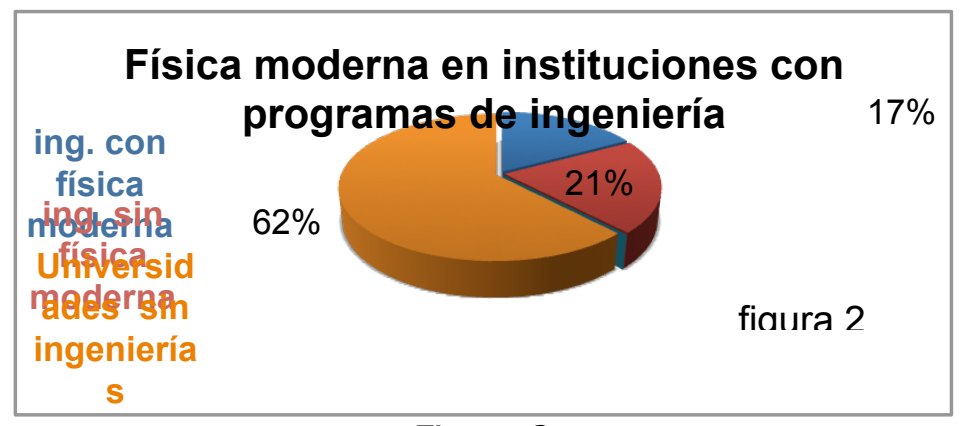

Figura 2

Igualmente, de este grupo de 42 universidades, las ingenierías de: Electrónica, eléctrica y mecánica se destacan no solo por estar entre las más ofertadas sino también porque superan en más del 50\% la enseñanza de la física moderna. Por ejemplo de las 17 
$5^{\circ}$ CONGRESO NACIONAL DE ENSEÑANZA DE LA FÍSICA.

Universidad Pedagógica Nacional (U. P. N)

Universidad Distrital Francisco José de Caldas (U. D. F. J. C)

Bogotá, Colombia. 16 al 20 de mayo 2011

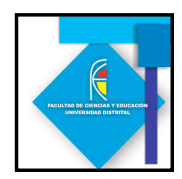

instituciones que ofrecen ingeniería electrónica, 10 de ellas tiene en su plan de estudio algún tópico de la física moderna, figura 3.

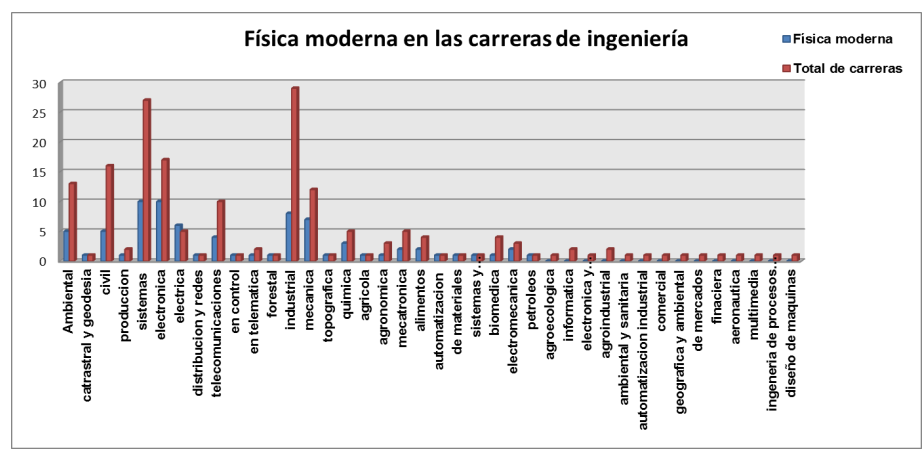

Figura 3

\section{La Importancia de la Física Moderna}

Los resultados de esta investigación muestran que la enseñanza de la física moderna tiene poca difusión en una de las poblaciones que se esperaría mayor conocimiento. Esta evidencia contrasta con National Academy of engineering en la publicación Educating the engineer of 2020 que dice "los avances recientes y emergente, como los de biotecnología, nanotecnología, tecnología de información y comunicaciones, ciencias de materiales y la fotónica, y otras tecnologías totalmente inesperadas serán uno de los cambios con los que la ingeniería y la enseñanza de la ingeniería tendrá que liderar hasta el 2020 y después"; también difiere con las exigencias para la acreditación de los programas de ingeniería según (ABET) Accreditation Board for Engineering and Technology de 2011- 2012, que se exponen en algunos de los criterios desarrollados por (ABET):

Criterio 3 objetivos educativos (i) El reconocimiento de la necesidad y la capacidad de involucrarse en un aprendizaje permanente.

(j) El conocimiento de temas de actualidad.

(k) La capacidad de utilizar las técnicas, habilidades y herramientas modernas de ingeniería necesarias para su comprensión y práctica.

Otra entidad como Unesco en declaración sobre la ciencia y el uso del saber científico dice. "La enseñanza científica, en sentido amplio, sin discriminación y que abarque todos los niveles y modalidades, es un requisito previo fundamental de la democracia y el desarrollo sostenible. En los últimos años se han tomado medidas en todo el mundo para promover la enseñanza básica para todos", igualmente importantes celebridades en el ámbito científico como León Lederman expone en iDetengan el siglo. No estamos preparados!, "Los más importantes descubrimientos del Siglo XX: la mecánica cuántica, la relatividad, la tectónica de placas, la Teoría de la Evolución de Darwin, la base molecular del ADN, la Teoría del Big Bang sobre el surgimiento y evolución del Universo, están insertados en estas tres columnas de la mente, materia y vida. Y el potencial para logros posteriores aparecerá cuando seamos capaces de combinar y concentrar estos baluartes". De lo anterior se puede concluir que para la formación académica de los estudiantes de ingeniería se requiere una base sólida de los 

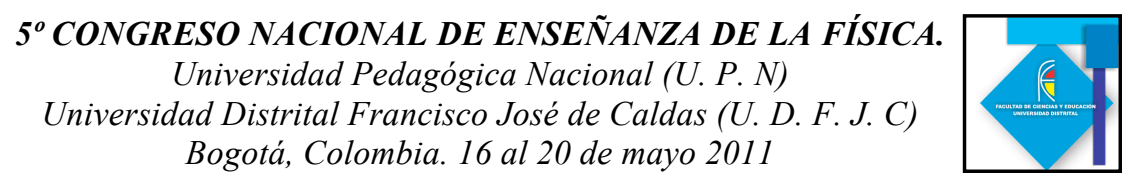

fundamentos físicos no solo clásicos sino también modernos, ya que estos rigen las tecnologías actuales.

Por ello vemos de vital importancia en la formación actual de los ingenieros tomar en cuenta estos requerimientos que fomentan la divulgación pertinente de las teorías modernas, es alarmante que a medida que avanza la tecnología la educación sigue acentuando en la formación de los ingenieros un pensamiento clásico sin posibilidad de competir en el diseño y desarrollo de tecnologías modernas que requieren los fundamentos físico dados en la teorías cuántica y relatividad.

Por supuesto quedan muchos interrogantes que responder:

¿Cuáles son las causas de la poca difusión de las ideas modernas de la física?

¿Hay profesores suficientemente preparados para enseñar significativamente las ideas modernas de la física?

¿Las directivas de las carreras de ingeniería muestran algún interés en la investigación que requiere las ideas modernas de la física?

¿Son las ideas de la física moderna "muy" elaboradas o complejas que su enseñanza es imposible?

\section{Referencias Bibliográficas}

Abet. Engineering Accreditation Commission (2011). Criteria for Accrediting Engineering Programs, www.abet.org.

Declaración de Budapest (1999).Marco general de acción de la declaración de Budapest, http:www.oei.org.co/cts/Budapest.dec.htm.

Dirección General del Catastro (2011).La Unidad Administrativa Especial de Catastro Distrital, http://www.catastro.meh.es/default.asp.

León, Lederman. ().iDetengan el siglo. No estamos preparados!, http://www.nodo50.org/ciencia popular/articulos/Lederman.htm

Ministerio de Educación Nacional (2011). Sistema Nacional de Información de la Educación Superior, $\quad$ http://www.mineducacion.gov.co/sistemasdeinformacion/1735/w3propertyname-2672.html

National Academy of engineering. (2005). Educating the engineer of 2020, Phase II, Committee on Engineering Education, National Academy of Engineering. 